\title{
High Power Flex-Propellant Arcjet Performance
}

\author{
Ron J. Litchford ${ }^{1}$ \\ NASA Marshall Space Flight Center
}

\begin{abstract}
A MW-class electrothermal arcjet based on a water-cooled, wall-stabilized, constricted arc discharge configuration was subjected to extensive performance testing using hydrogen and simulated ammonia propellants with the deliberate aim of advancing technology readiness level for potential space propulsion applications. The breadboard design incorporates alternating conductor/insulator wafers to form a discharge barrel enclosure with a $2.5-\mathrm{cm}$ internal bore diameter and an overall length of approximately 1 meter. Swirling propellant flow is introduced into the barrel, and a DC arc discharge mode is established between a backplate tungsten cathode button and a downstream ringanode/spin-coil assembly. The arc-heated propellant then enters a short mixing plenum and is accelerated through a converging-diverging graphite nozzle. This innovative design configuration differs substantially from conventional arcjet thrusters, in which the throat functions as constrictor and the expansion nozzle serves as the anode, and permits the attainment of an equilibrium sonic throat (EST) condition. During the test program, applied electrical input power was varied between 0.5-1 MW with hydrogen and simulated ammonia flow rates in the range of 4-12 g/s and 15-35 g/s, respectively. The ranges of investigated specific input energy therefore fell between 50-250 MJ/kg for hydrogen and 10-60 MJ/kg for ammonia. In both cases, observed arc efficiencies were between 40-60 percent as determined via a simple heat balance method based on electrical input power and coolant water calorimeter measurements. These experimental results were found to be in excellent agreement with theoretical chemical equilibrium predictions, thereby validating the EST assumption and enabling the utilization of standard TDK nozzle expansion analyses to reliably infer baseline thruster performance characteristics. Inferred specific impulse performance accounting for recombination kinetics during the expansion process implied nearly frozen flow in the nozzle and yielded performance ranges of 800-1100 sec for hydrogen and 400-600 sec for ammonia. Inferred thrust-to-power ratios were in the range of 30-10 lbf/MW $/ \mathbf{M W}_{\mathrm{e}}$ for hydrogen and $\mathbf{6 0 - 2 0} \mathrm{lbf} / \mathbf{M W}_{\mathrm{e}}$ for ammonia. Successful completion of this test series represents a fundamental milestone in the progression of high power arcjet technology, and it is hoped that the results may serve as a reliable touchstone for the future development of MW-class regeneratively-cooled flex-propellant plasma rockets.
\end{abstract}

$\begin{array}{ll}A & =\text { area } \\ d & =\text { diameter } \\ e & =\text { electron charge } \\ F & =\text { thrust } \\ g_{0} & =\text { sea-level gravitational acceleration } \\ I & =\text { current } \\ \text { Isp } & =\text { specific impulse } \\ j & =\text { current density } \\ k & =\text { Boltzmann constant } \\ \dot{m} & =\text { mass flow rate } \\ p & =\text { pressure }\end{array}$

\section{Nomenclature}

$$
\begin{array}{ll}
P_{e}, P_{j e t} & =\text { electrical or jet-kinetic power } \\
Q & =\text { thermal power } \\
q^{\prime \prime} & =\text { heat flux } \\
T & =\text { temperature } \\
u_{e} & =\text { exhaust velocity } \\
V & =\text { voltage } \\
\eta & =\text { efficiency } \\
\varphi_{e}=P_{e} / \dot{m} & =\text { specific input energy } \\
\phi & =\text { work function } \\
\Pi & =\text { Peltier coefficient } \\
\rho & =\text { density }
\end{array}
$$

\footnotetext{
${ }^{1}$ Principal Investigator, OCT / Game Changing Development Program Office, Associate Fellow AIAA.
} 


\section{Introduction}

$\mathrm{E}$ LECTROTHERMAL arcjet thrusters represent a relatively mature space propulsion technology with developmental roots extending back to pioneering efforts at Giannini Scientific Corp. and AVCO Corp. ${ }^{1-3}$ Continued development over subsequent decades has sustained technical progression with flight qualification of a low-power north-south station-keeping (NSSK) hydrazine arcjet occurring in 1991 followed by a launch on Telstar4 in $1993 .{ }^{4}$ Since then utilization has become routine, and more than 50 satellites currently in orbit incorporate 1-2 $\mathrm{kW}$ arcjet thrusters as a means of improving propellant utilization efficiency. The desire for fast and efficient orbital transfer, however, compels an extension of power level to the $30-50 \mathrm{~kW}$ range, ${ }^{5}$ and to address this need, the USAF/AFRL subsequently developed and executed the Electric Propulsion Space Experiment (ESEX) as a flight demonstration of a $26-\mathrm{kW}$ ammonia arcjet for satellite applications. ${ }^{6}$ Beyond this medium power range, interest has been limited since there are no critical high-power mission drivers for commercial or military satellite usage in earth orbit, and thus little practical incentive for further development. With renewed interest in exploration missions beyond earth orbit, however, and the potential availability of high electric power supplies in space based on large solar arrays or nuclear reactors, there has been a revitalization of interest in higher power electric thrusters, particularly technologies capable of yielding high specific impulse at high thrust levels. Thus, for large robotic or manned interplanetary missions, to Mars for example, multi-megawatt nuclear electric propulsion (NEP) systems are under active study as a potentially superior alternative to nuclear thermal propulsion (NTP). ${ }^{7}$

Despite the obvious storage difficulties, hydrogen is a highly desirable propellant choice because it yields the highest possible performance characteristics. Moreover, hydrogen has an attractive specific heat capacity and excellent potential for designs employing regenerative heat transfer. Based on such practical considerations, substantial work toward the development of high-power hydrogen arcjets has previously been undertaken with some ground based demonstrations extending above $100 \mathrm{~kW} .^{8-12}$ It should be noted, however, that many space mission scenarios do not necessarily require the performance potential associated with hydrogen and could be entirely feasible with ammonia or hydrazine thereby avoiding highly demanding cryogenic fluid management challenges. The objective of this work was to empirically examine the performance of an innovative flex-propellant electrothermal arcjet using both hydrogen and simulated ammonia over a power range of $0.5-1 \mathrm{MW}_{\mathrm{e}}$ with the deliberate aim of advancing the technology readiness level for potential space propulsion applications.

Multi-MW electric arc plasma generation technology has been in existence for decades, of course, but these facilities are typically operated with air in support of high-speed aerodynamic research and thermal protection system development. There are a few past examples of high-power arcjet systems with demonstrated flex-propellant capability, such as Aerojet's 1-MW multi-gas Hyperthermal Environment Simulator used extensively in support of rocket materials development and qualification, ${ }^{13}$ but there does not appear to be any open literature data delineating the performance characteristics obtained with this particular device. Some useful hydrogen performance data has also been reported for a 250-kW Huels-type device (Linde N-250) operated by the McDonnell Douglas Corp., but this design configuration is not conducive to propulsion implementations and therefore of limited value. ${ }^{14}$

This paper presents the results of recently completed experiments using a MW-class water-cooled segmentedconstrictor type multi-gas arcjet and supporting analysis pertinent to the advancement of technology readiness level. Completion of these demonstration tests represents a fundamental milestone in the technical progression toward higher powers, and it is hoped that the results may serve as a constructive basis for the future development of MWclass regeneratively-cooled flex-propellant plasma rockets.

\section{Arcjet Configuration and Operation}

The arcjet hardware used in this experimental program is a $1-\mathrm{MW}_{\mathrm{e}}$ (nominal) multi-gas segmented device, which operates in a wall stabilized constricted arc DC discharge mode. A cross-sectional assembly schematic is shown in Fig. 1. This particular device has a $2.5-\mathrm{cm}$ internal bore diameter and follows the traditional segmentation design philosophy whereby alternating conductor/insulator wafers are stacked together to form the full length assembly. The heat conducting copper segments are water-cooled and are separated by boron nitride insulators in stacked pack subassemblies, which are held securely together by four Inconel-718 tie rods. These subassembly packs are then attached in a sequential manner to form the full arc-heater column, which spans an overall length of about 1 meter in the three-pack configuration shown. The working gas is injected tangentially through four choked orifice jets in a Primary Gas Injection (PGI) segment near the rear of the arc-heater, and a DC arc discharge is established between a thoriated tungsten cathode button in the rear sealing flange and a copper anode ring at the arc-heater exhaust. A magnetic spin coil is located around the anode ring to induce continuous rotation of the arc attachment point. A high-density graphite nozzle assembly is directly attached to the arcjet anode flange. It is important to note that this

American Institute of Aeronautics and Astronautics 
configuration differs substantially from the conventional arcjet thruster design approach in which the throat functions as a constrictor and the expansion nozzle serves as the anode. Here, the arc extinguishes on the ring anode upstream of the throat and a short nozzle contraction section provides a region for mixing and equilibration. This arrangement necessarily increases convective wall losses; however, it also greatly simplifies theoretical interpretation of the nozzle expansion process.

Global calorimeter characteristics are deduced from measurements of total cooling

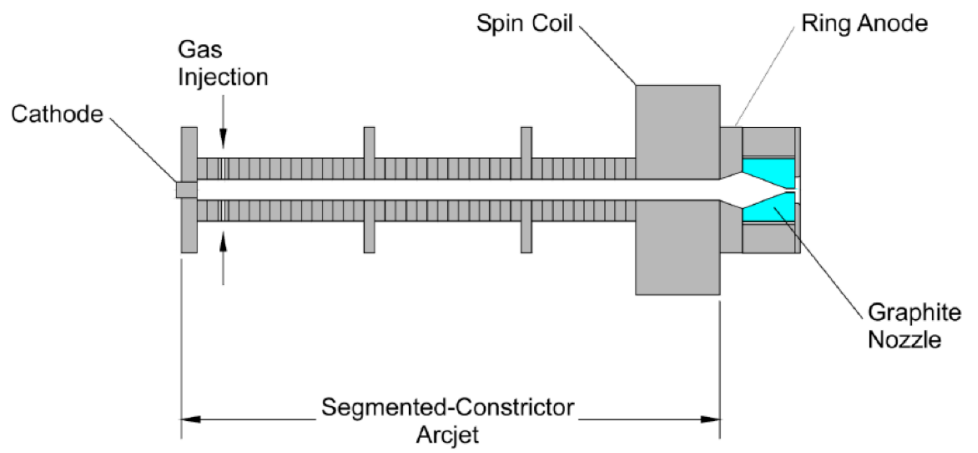

Figure 1. Cross-sectional assembly schematic of MW-class, water-cooled, segmented-constrictor type arcjet.

water flow rate and associated temperature

rise across the device. Because the variation in heat transfer along the device is also of great practical interest, individual flow rate and temperature rise measurements were incorporated into selected cooling circuits to provide localized calorimetry and component heat flux estimations. These individual calorimeter circuits are identified in the detailed arcjet layout shown in Fig. 2 and include the cathode, three barrel segments, the anode, and the anode transition.

In order to start the device at atmospheric chamber pressure, a modest flow of argon is first introduced into the arc-heater and an initial gas discharge is established between the cathode button and a starting segment, which is located just downstream of the PGI segment. The anode power lead is then automatically switched from the starting segment to the anode ring by opening a vacuum contactor, which establishes a stable arc down the full length of the arc-heater. At this point, argon flow can be replaced with the desired working gas and the system can be ramped to the desired operating state. Propellant flow rate is set supply pressure and measured via subsonic venturi. In the case of simulated ammonia, the propellant is manufactured by combining separate hydrogen and nitrogen flow streams with the appropriate mole fraction characteristics prior to injection.

The arcjet is energized by a saturable reactor DC power supply that can sustain a continuous operating power of $0.75-\mathrm{MW}_{\mathrm{e}}$ on an indefinite basis and can deliver an intermittent power burst of $1.5-\mathrm{MW}_{\mathrm{e}}$ for 5 to 10 minutes. This power supply can be configured in either parallel mode (2500 volts open circuit) or series mode (5000 volts open circuit) as needed to match the internal impedance characteristics of the gas discharge. To accommodate the high impedance characteristics of hydrogen and ammonia propellants, the power supply was operated in series mode configuration during all tests.

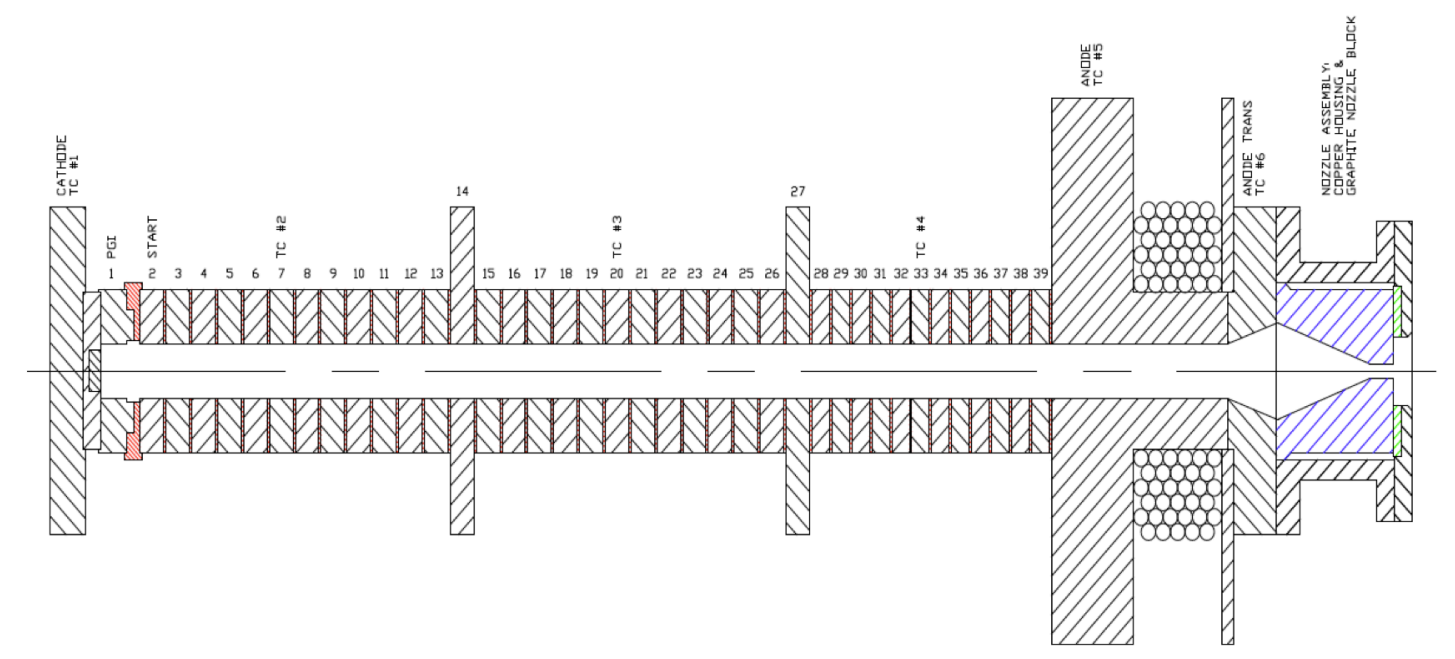

Figure 2. Detailed arcjet layout with individual calorimeter circuits identified: 1 - cathode; 2 - barrel segment \#7; 3 - barrel segment \#20; 4 - barrel segment \#33; 5 - anode; 6 - anode transition. 


\section{Performance Characterization}

\section{A. Steady-State Performance Attributes}

Two series of arcjet performance tests were conducted using hydrogen and simulated ammonia propellants. During this test program, applied electrical input power was varied between 0.5-1 MW with hydrogen and simulated ammonia flow rates in the range of 4-12 g/s and $15-35 \mathrm{~g} / \mathrm{s}$, respectively. The ranges of investigated specific input energy therefore fell between 50-250 MJ/kg for hydrogen and 10-60 MJ/kg for ammonia. Following conventional practice, specific input energy, $\varphi_{e}$, is defined as the ratio of the applied electrical power, $P_{e}$, to the propellant mass flow rate, $\dot{m}$ :

$$
\varphi_{e}=\frac{P_{e}}{\dot{m}}
$$

The two controllable parameters in these tests were the applied arc current, as governed by the saturable reactor set-point current, and the propellant mass flow rate, as established by the gas supply pressure regulator. Nozzle size was selected to maintain a chamber pressure range of $5-15$ bar in both test series. In the case of hydrogen, nominal throat diameters of $0.278^{\prime \prime}$ and $0.238^{\prime \prime}$ were needed to accommodate the very wide range of specific input energies. For simulated ammonia, a nominal throat diameter of $0.350^{\prime \prime}$ was adequate for all tests.

During each test, basic operating parameters such as current, voltage, chamber pressure, hydrogen mass flow rate, cooling water flow rate, and cooling water temperature rise were recorded from which we could infer system power balance and electrical-to-thermal conversion efficiency. Examination of this test data verified that steadystate operational conditions could be reliably established within $\sim 20$ seconds of arc initiation and facility ramp-up, as may be seen from the start-up transients shown in Fig. 3 for a typical 60 -sec hydrogen run at $750-\mathrm{kW}_{\mathrm{e}}$. Steady-state performance measurements were obtained by time averaging the data over the final $1 / 2$-second of each test record.

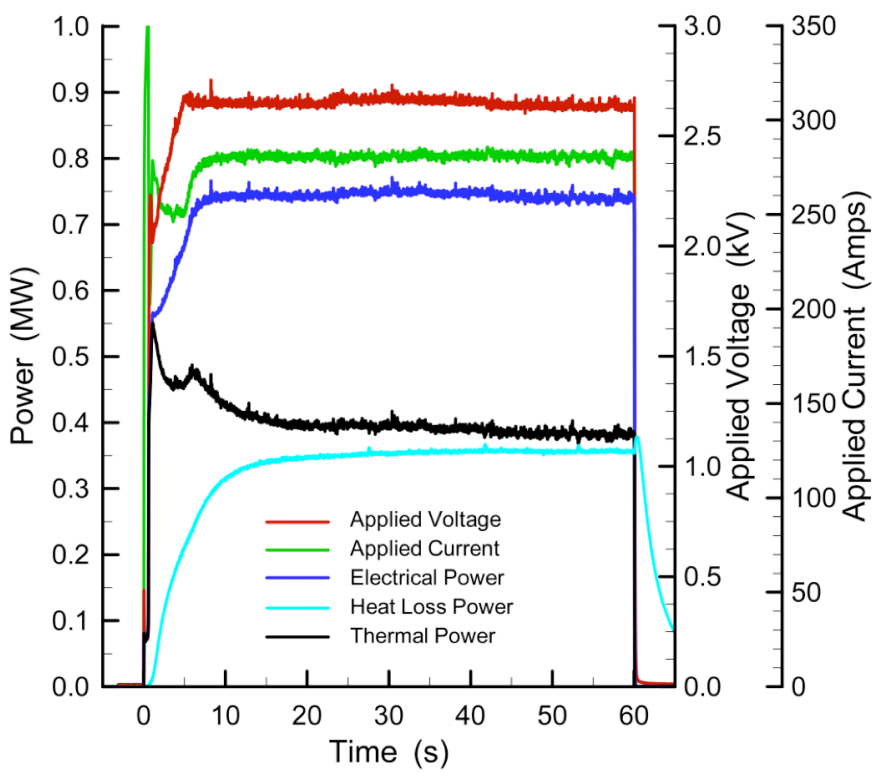

Figure 3. Typical arcjet startup transients at $750-\mathrm{kW}_{\mathrm{e}}$.

A key performance parameter of interest for any electric propulsion system is the thrust efficiency, $\eta_{t}$, defined as the ratio of the exhaust jet power to electrical input power:

$$
\eta_{t}=\frac{P_{j e t}}{P_{e}}=\frac{\dot{m} u_{e}^{2} / 2}{P_{e}}=\frac{u_{e}}{2} \frac{F}{P_{e}}=1 / 2 g_{0} I s p\left(\frac{F}{P_{e}}\right)
$$

where $u_{e}$ is the exhaust velocity, $F$ is the thrust, Isp is the specific impulse, and $g_{0}$ is the sea-level gravitational acceleration. All practical thrusters depart from ideal performance due to the compounded effect of various process inefficiencies, and this nonideality may be catalogued by defining a partial efficiency for each major contributing factor. For electrothermal thrusters, the important partial efficiencies include the arc efficiency, $\eta_{a}$, the frozen flow efficiency, $\eta_{f}$, and the aerodynamic/viscous efficiency, $\eta_{v}$, such that the thrust efficiency takes the form:

$$
\eta_{t}=\eta_{a} \eta_{f} \eta_{v}
$$

The compounding effects of these process inefficiencies on overall thruster performance are depicted by the process diagram shown in Fig. 4 where the factor $1-\eta_{a}$ accounts for energy losses in the arc heating process, $1-\eta_{f}$ accounts for the non-recoverable internal energy of the exhaust flow, and $1-\eta_{v}$ accounts for the viscous and aerodynamic profile losses throughout the nozzle expansion process. 


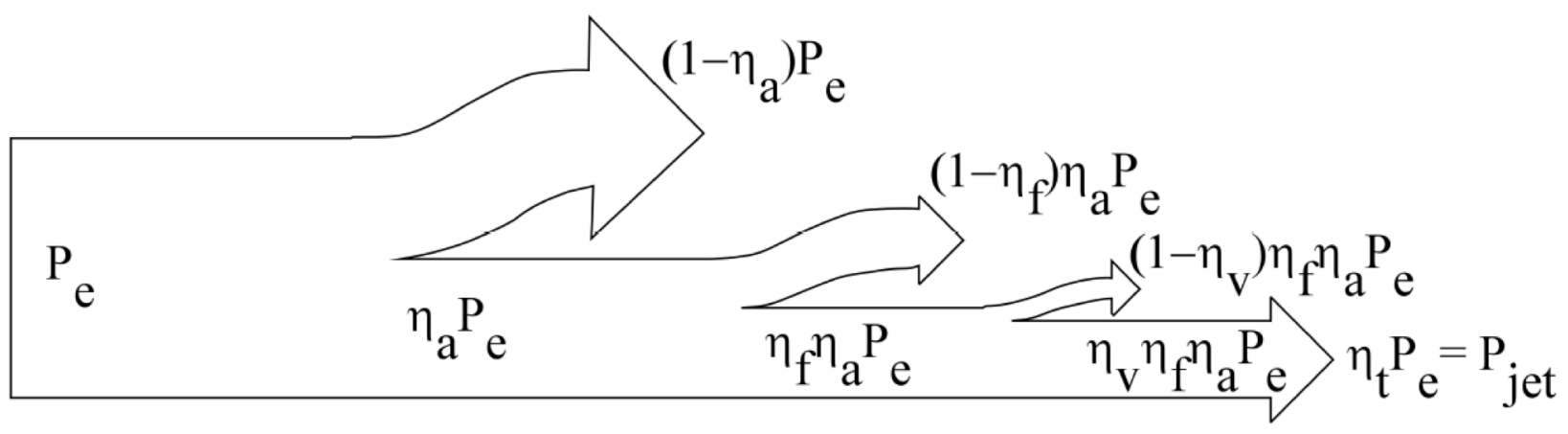

Figure 4. Illustration of electrothermal thruster process inefficiencies and compounding effect on overall thrust efficiency.

Deduction of the arc efficiency from steady-state performance data is straightforward and follows directly from its definition as the ratio of total thermal power delivered to the working fluid, $Q_{\text {gas }}$, to the total applied electrical power, $P_{e}$ :

$$
\eta_{a}=\frac{Q_{\text {gas }}}{P_{e}}=\frac{P_{e}-Q_{\text {loss }}}{P_{e}}
$$

where the heat loss thermal power, $Q_{\text {loss }}$, is derived from cooling water calorimeter measurements. Evaluation of the frozen flow and aerodynamic/viscous efficiencies requires a more elaborate analysis procedure and will be discussed at length below.

It is of considerable interest to examine arc efficiency in some detail at this point since it is these characteristics that determine total gas enthalpy available for thrust production at the nozzle entrance. Empirically derived arc efficiencies for the hydrogen and simulated ammonia test series are shown in Figs. 5 and 6, respectively, from which we observe a strong correlation with applied electrical power level and a weaker but non-negligible dependence on chamber pressure, $p_{c}$. Thus, accurate correlation of the data sets require bilinear fits accounting for both power and pressure effects in the form:

$$
\eta_{a}^{(f i t)}=A+B P_{e}+C p_{c}
$$

Comparisons between the measured and bilinear fits indicate fidelity of $\pm 1 \%$ over the entire data set.

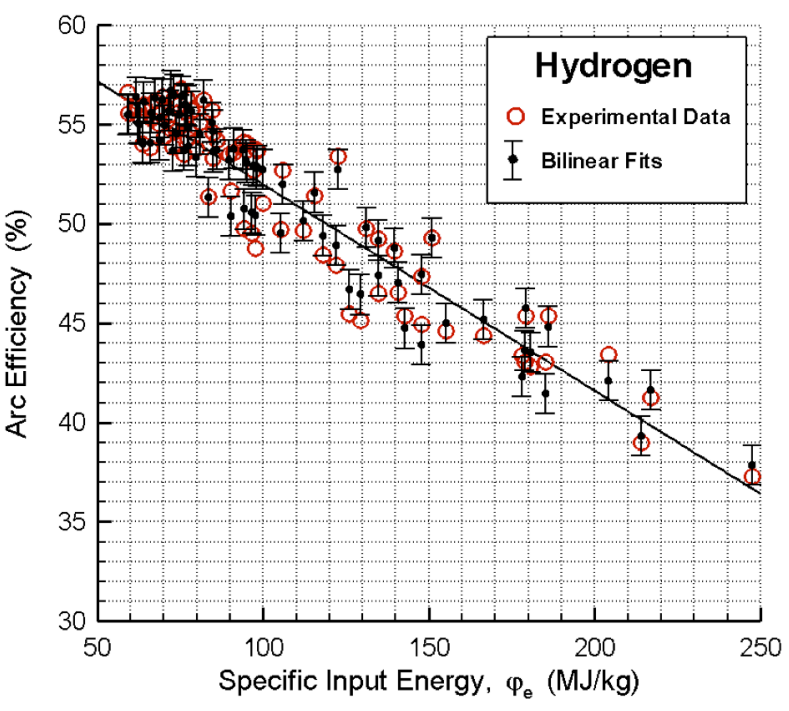

Figure 5. Hydrogen arc efficiency characteristics.

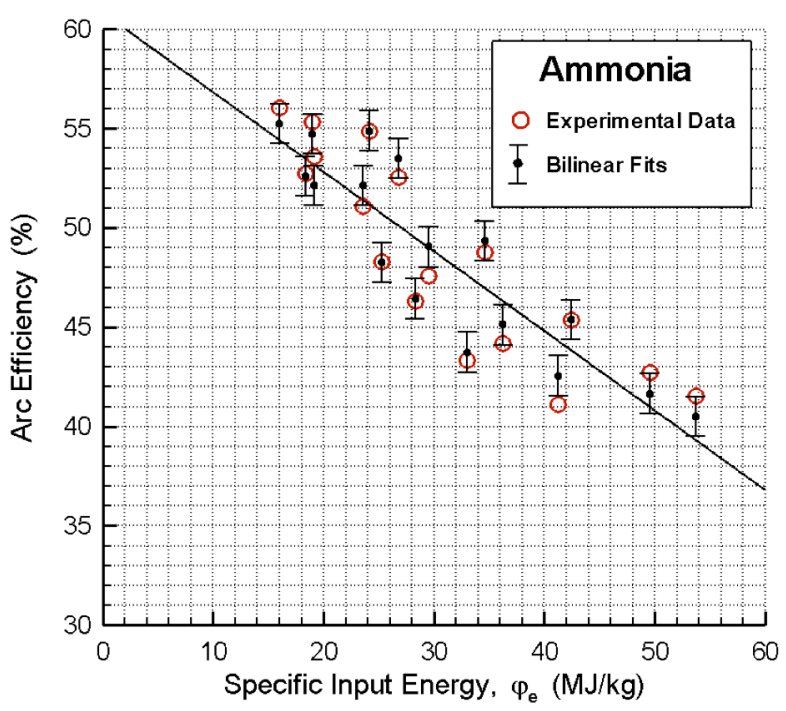

Figure 6. Ammonia arc efficiency characteristics.

American Institute of Aeronautics and Astronautics 


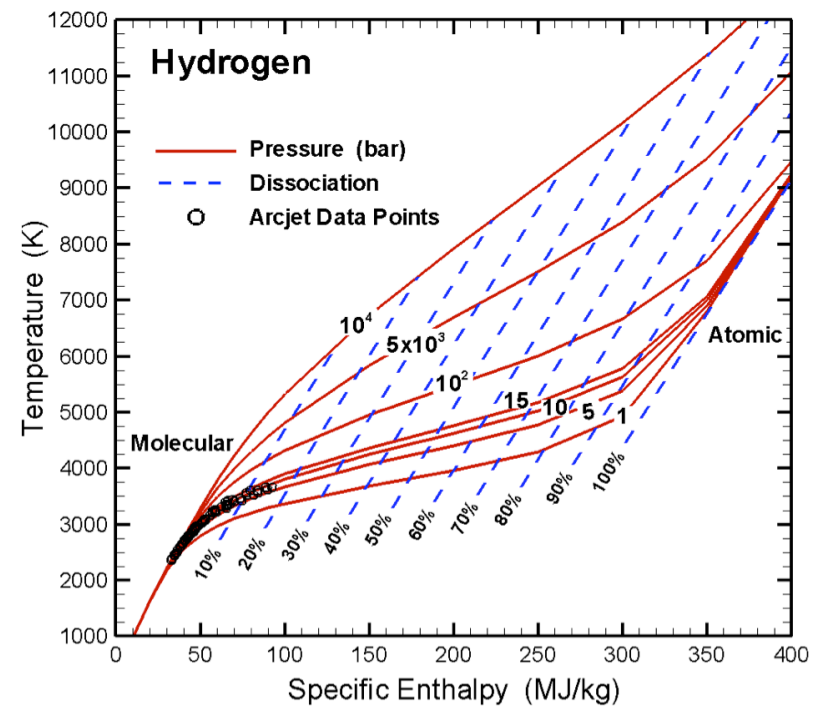

Figure 7. Hydrogen thermodynamic state map.

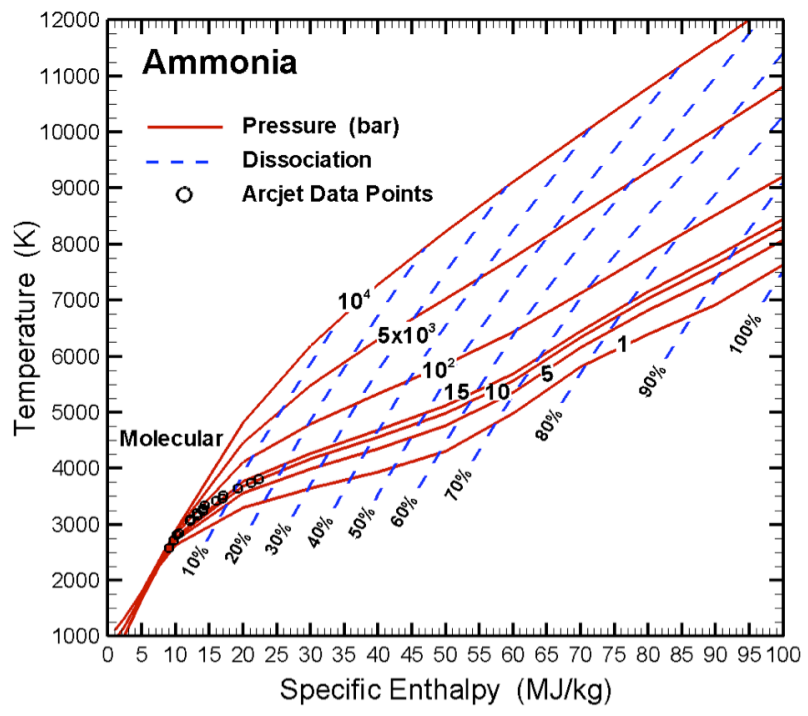

Figure 8. Ammonia thermodynamic state map.

By assuming thermodynamic equilibrium and exercising the NASA Chemical Equilibrium Analysis (CEA) code, it is possible to construct thermodynamic state maps for these propellants yielding important insight into the degree of internal energy bound up by dissociation processes. Figures 7 and 8 , for instance, depict pressure and dissociation fraction contours on the plane of specific enthalpy, $Q_{g a s} / \dot{m}$, versus total chamber temperature, $T_{0}$, for hydrogen and ammonia, respectively.

Implied state points for all test measurements are also shown on these maps to illustrate the empirical regime of investigation. These plots clearly demonstrate the strong suppression of temperature, and therefore thruster performance, with increasing specific enthalpy due to the large amounts of energy absorbed by dissociation. Because dissociation is favored at lower pressure, it is equally obvious that operation at higher chamber pressures would yield natural performance benefits though practical system level considerations constrain the achievable. Such large internal mode dissociation energy at the nozzle entrance is highly problematic in that recombination tends to be slow and may be expected to lag behind its equilibrium level thereby contributing to significant fozen flow losses.

\section{B. Equilibrium Sonic Throat}

By introducing a small plenum region between the ring anode and nozzle throat, the highly nonuniform flow exiting the discharge barrel is provided with time to mix and equilibrate. This is in contrast to modern arcjet design practice in which the throat functions as the constrictor section and the nozzle expansion surface serves as the anode. The more compact modern design approach is known to be very effective at minimizing losses but it also greatly complicates thruster performance analysis. ${ }^{15}$ This is not the case for the current design configuration, however, where analysis of the experimental data supports the existence of an equilibrium sonic throat (EST) condition. ${ }^{16}$

For instance, given the empirically deduced thermal flow power, $Q_{\text {gas }}$, it is possible to infer a pseudo heat of combustion that can then be used in the NASA Chemical Equilibrium Analysis (CEA) code to predict the effective equilibrium thrust chamber conditions for fixed throat diameter, $d_{t}$. Forcing the calculation to match the measured chamber pressure, thus yields a choked EST mass flow rate, $\dot{m}^{(E S T)}$, which can then be checked against the experimentally determined value, $\dot{m}^{(\text {(exp) }}$. The results of this exercise for the ratio $\dot{m}_{H_{2}}^{(\exp )} / \dot{m}_{H_{2}}^{(E S T)}$ are summarized in Fig. 9 demonstrating excellent agreement over the complete data set thereby confirming validity of the EST assumption under all test conditions. The availability of the EST condition at the nozzle throat is extremely significant in that it provides a solid basis for estimating nozzle/thrust performance.

Given the fact that dissociation plays such an important role, one may naturally question how well performance characteristics derived from simulated ammonia operation can be used as a basis of representation for true ammonia operation. A simple and effective check on this hypothesis can now be examined using the established EST conditions by computing the ratio $T_{0, S \text { Simulated } H_{3}}^{(E E T)} / T_{0, N H_{3}}^{(E E T)}$. The results of this exercise are summarized in Fig. 10 demonstrating very good agreement and thereby substantiating validity. 


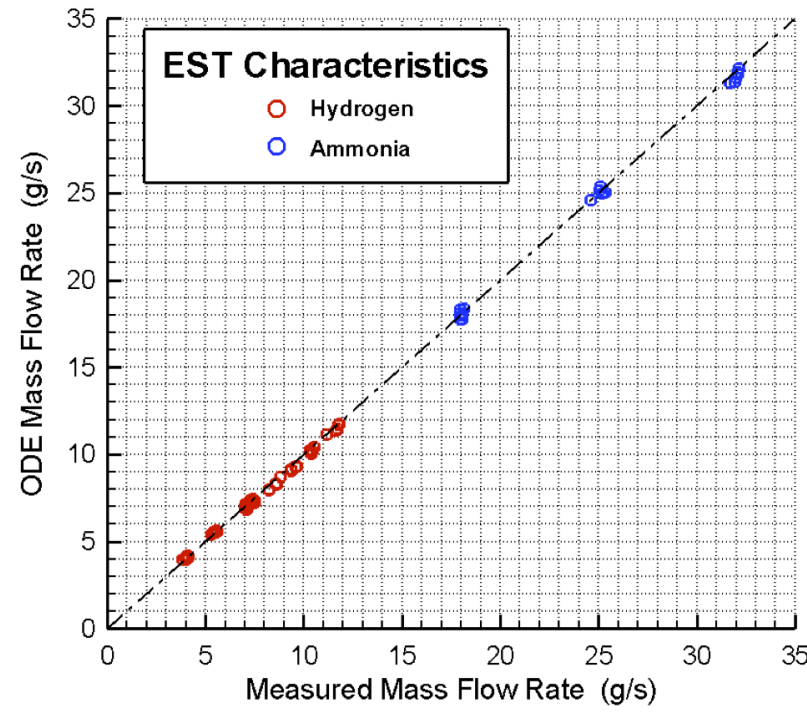

Figure 9. Combined EST characteristics.

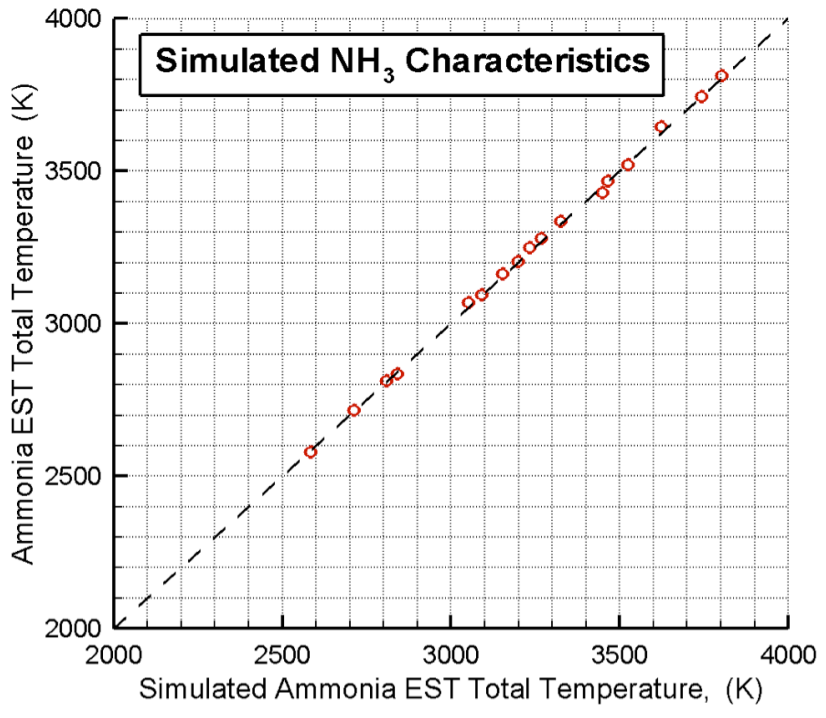

Figure 10. Simulated ammonia characteristics.

\section{Inferred Thrust Performance}

Because the device was operated against atmospheric backpressure, it was not possible to expand the flow and measure thruster performance. However, the existence of EST conditions offers the possibility of inferring thrust performance characteristics using standard nozzle expansion analysis tools, such as Sierra Engineering's Two Dimensional Kinetic (TDK) code. TDK performs a 2D nonequilibrium nozzle performance calculation using extensively validated reaction kinetics and includes boundary layer effects. By starting a calculation from the arcjet EST conditions it is therefore possible to reliably estimate in-space thruster performance with a high degree of confidence.

As a standard basis for comparison, TDK analyses were carried out for all test cases assuming EST thrust chamber conditions for a $20^{\circ}$ half-angle conical nozzle with 200:1 area expansion ratio and adiabatic boundary layer. As part of the analysis, TDK also computes performance predictions corresponding to one-dimensional frozen (ODF) and one-dimensional kinetic (ODK) expansion processes. Computed results for Isp ${ }^{(\mathrm{ODF})}$ and Isp ${ }^{(\mathrm{ODK})}$ are presented in Figs. 11 and 12 as a function of specific input energy and the close agreement clearly demonstrates that recombination kinetics is largely suppressed throughout the expansion process implying nearly frozen flow and unavoidable performance limitations for both propellants. The results for Isp ${ }^{(\mathrm{TDK})}$ yield even lower performance due to inclusion of aerodynamic/viscous loss mechanisms. As a result, inferred specific impulse performance predictions accounting for slowed recombination kinetics yielded performance ranges of 800-1100 sec for hydrogen and $400-600 \mathrm{sec}$ for ammonia.

An additional performance parameter of key interest is the arcjet thrust-to-power ratio, also presented in Figs. 11 and 12 as a function of specific input energy. Evaluation of this parameter follows from the TDK computed thrust and the measured steady-state electrical input power. Inferred thrust-to-power ratios were in the range of 30-10 $\mathrm{lbf} / \mathrm{MW}_{\mathrm{e}}$ for hydrogen and $60-20 \mathrm{lbf} / \mathrm{MW}_{\mathrm{e}}$ for ammonia. These figures stand as direct refutation to the commonly held misconception regarding electric thrusters as inherently unscalable for high thrust operation. The key constraining factor for high-thrust electric propulsion is not thruster scalability but the non-availability of low specific mass power generation systems.

An often-posed question is why does thrust efficiency tend to decline as specific impulse increases? The answer, of course, is associated with increasing process inefficiencies with increasing specific input energy. But the underlying factors can be difficult to discern. To generate some insight into such questions, it is useful to exploit the available TDK performance predictions as a basis of for estimating the frozen flow and aerodynamic/viscous process efficiencies in addition to the over all thrust efficiency. For instance, estimates for frozen flow efficiency independent of aerodynamic profile and wall losses may be deduced from ODK predictions, which then provide a basis for separating the aerodynamic/viscous efficiency based on TDK calculations including boundary layer effects. The full compliment of process efficiencies derived from this procedure are presented as a function of specific input energy in Figs. 13 and 14 for hydrogen and ammonia, respectively. 


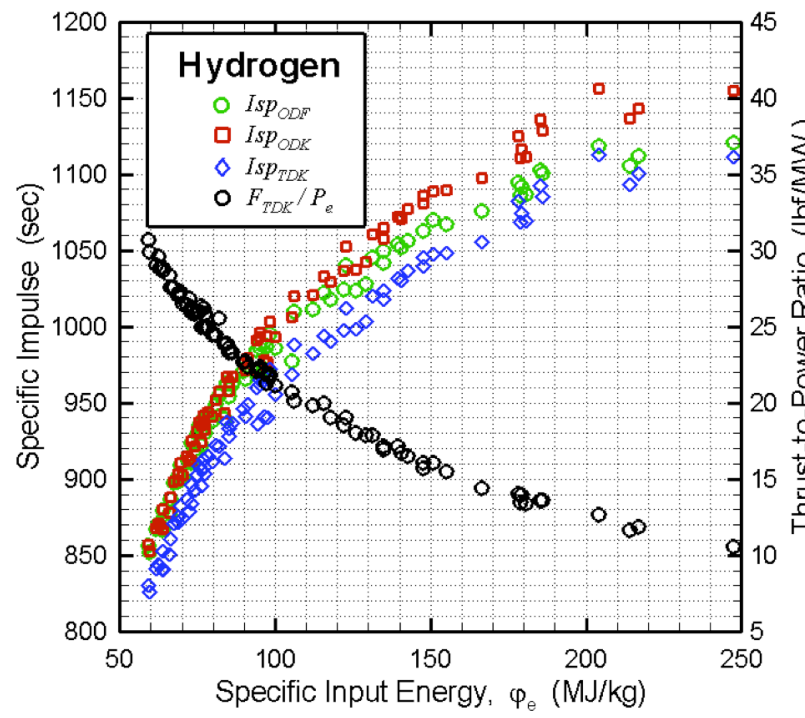

Figure 11. Hydrogen performance characteristics.

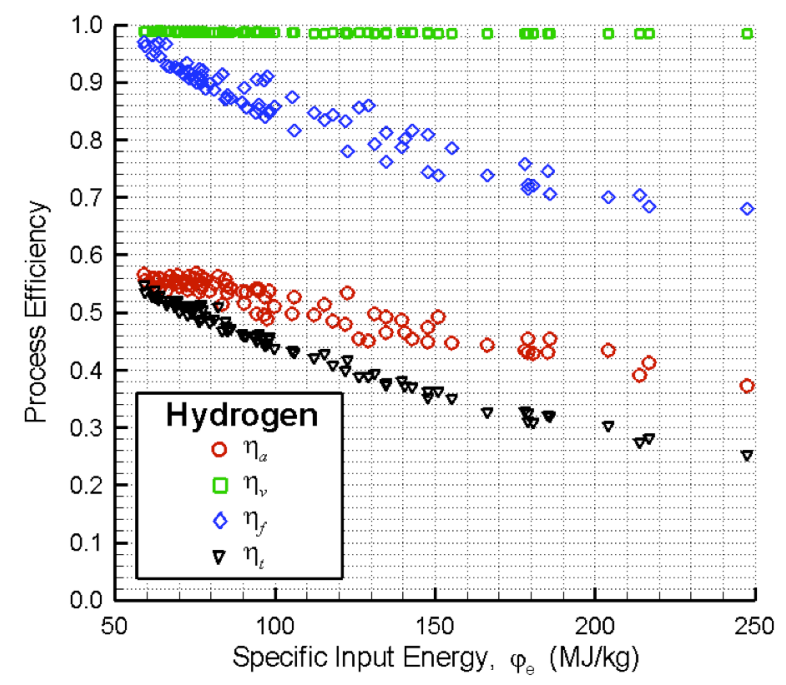

Figure 13. Hydrogen efficiency characteristics.

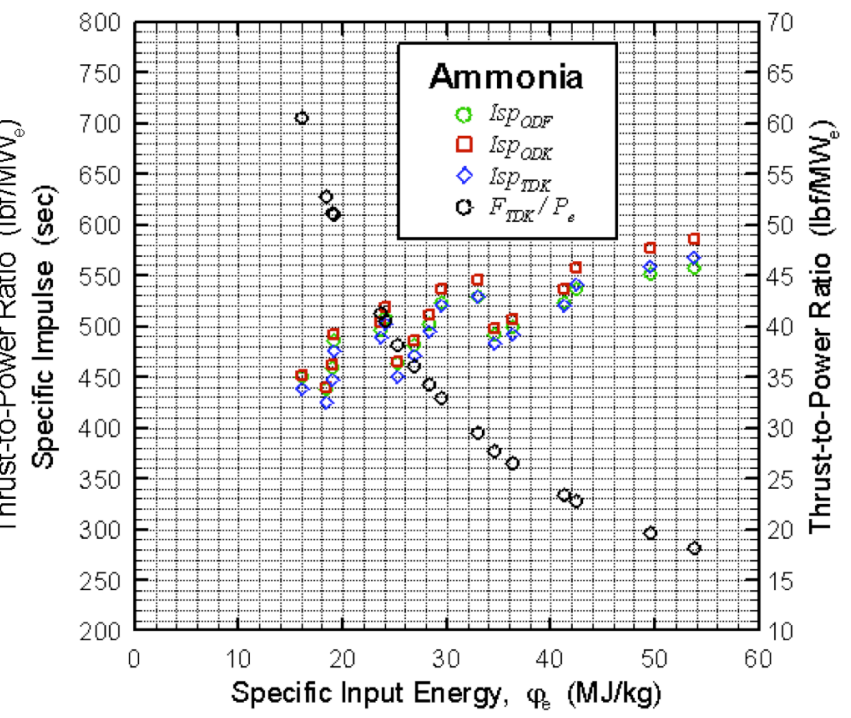

Figure 12. Ammonia performance characteristics.

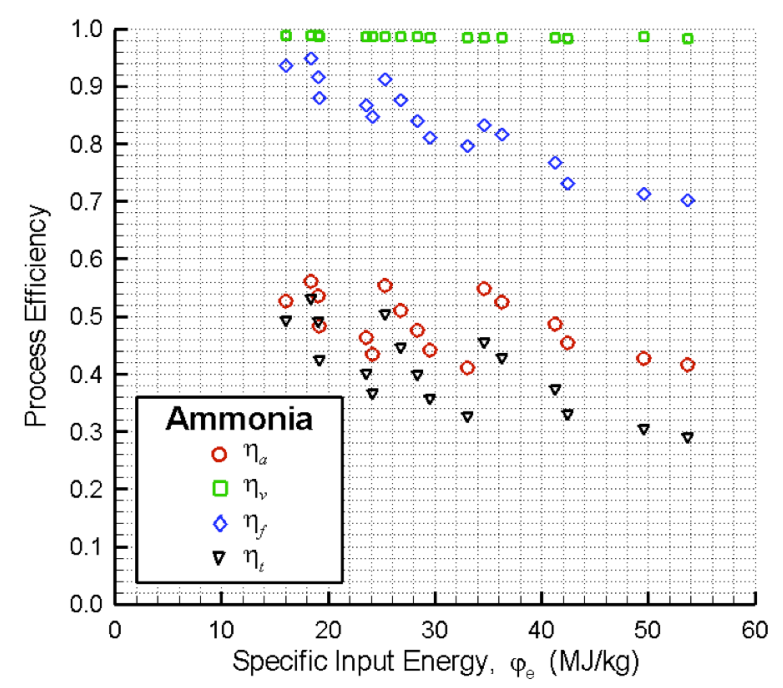

Figure 14. Ammonia efficiency characteristics.

These plots clarify the various inefficiency contributions and indicate that the most significant decrements are associated with electric-to-thermal conversion in the arc heating process and frozen flow losses. As the current and plasma temperature rise with increasing applied power, for instance, convection/radiation heat transfer must increase at the same time that plasma impedance and electric-to-thermal coupling are declining. The associated drop in arc efficiency is an unavoidable, intrinsic feature of any arc-heated process and imposes a point of diminishing returns for high power arcjet technology. Furthermore, as the plasma is heated ever higher, more and more energy is locked up by internal mode dissociation and effective recovery during the nozzle expansion process becomes seriously impeded by slowed recombination kinetics. Due to these effects, $\eta_{a}$ exhibits a decline of $60-40 \%$ as $\eta_{f}$ declines over the range of $98-70 \%$ for both hydrogen and ammonia. Aerodynamic profile and viscous losses tend to be minor and relatively flat with $\eta_{v}$ in the range of $98-99 \%$. The combined effect of these efficiency characteristics yield thrust efficiencies in the range of $56-28 \%$ for hydrogen and $50-30 \%$ for ammonia over the specific input energy range of investigation. 


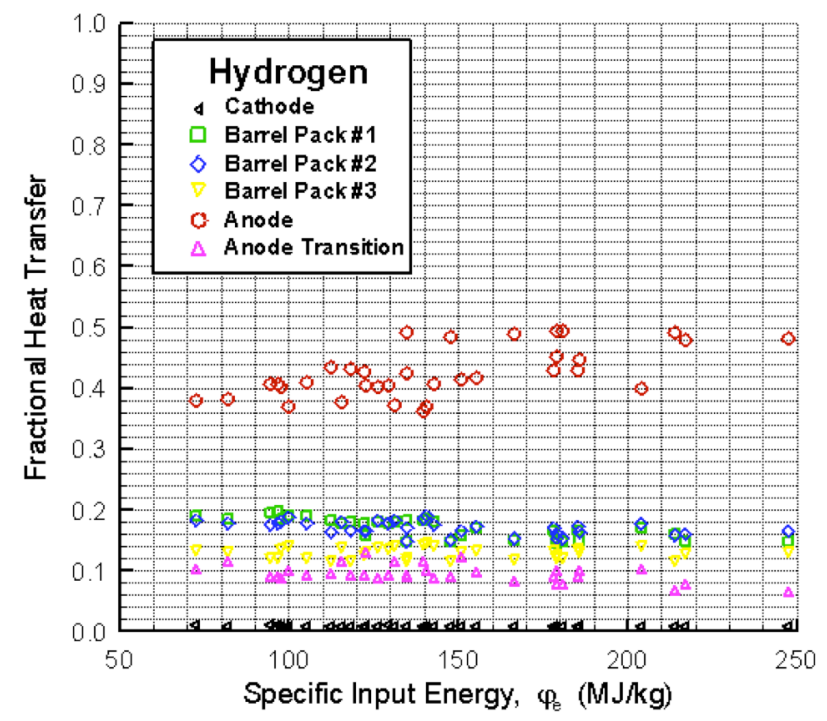

Figure 15. Hydrogen heat transfer characteristics.

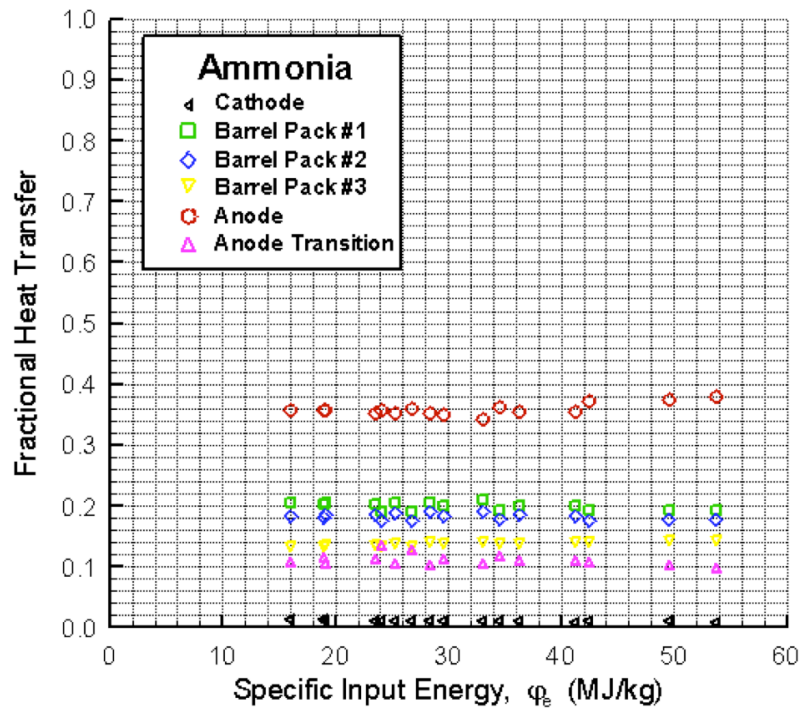

Ammonia heat transfer characteristics.

\section{Heat Transfer Characteristics}

Variations in heat transfer along the device are of great practical interest from a practical engineering standpoint for thermal management and optimization of regenerative cooling strategies. While the simple global heat loss measurement is adequate for determining the all important arc efficiency parameter, calorimetry measurements on individual cooling circuits provide important localized component heat flux estimations. For this purpose, a selected number of independent cooling circuits were instrumented for flow rate and temperature rise during both test series. These circuits are identified in the detailed arcjet layout of Fig. 2 and include the cathode, three separate barrel segments, the anode, and the anode transition. The results of these measurements are displayed as the fractional heat transfer for each major component subsection in Figs. 15 and 16 for hydrogen and ammonia, respectively.

In both cases, these results indicate that the cathode only absorbs about $1 \%$ of the total heat loss while the cooling segment packs display monotonically decreasing heat absorbtion in the range of $20-10 \%$ along the barrel length. As expected, the anode carries the highest heat load with a range of 35-50\% during hydrogen testing and 35$40 \%$ during ammonia testing. The diverging anode transition section absorbed between $8-12 \%$ of the total heat loss in both cases. Contributing effects to anode heat loading include ohmic dissipation within the metal and the heat delivered to the anode surface, which may be expressed in the following form:

$$
q_{\text {anode }}^{\prime \prime}=q_{\text {conv }}^{\prime \prime}+q_{\text {rad }}^{\prime \prime}+j / e\left(\phi_{\text {anode }}+2 k T_{\text {e,anode }}-e \Pi_{\text {anode }}\right)
$$

Here, $j$ is the electron current density, $e$ is the electron charge, $\phi_{a n o d e}$ represents the potential energy equal to the anode work function where the Fermi energy level has been neglected, $k$ is the Boltzmann constant, $T_{e, \text { anode }}$ is the electron temperature at the anode surface, and $\Pi_{\text {anode }}$ is the Peltier coefficient for the anode.

\section{E. Hardware Assessments}

In general, the water-cooled arcjet hardware displayed excellent durability, reliability, and long-life operational capability. Post-test inspections of the components indicated normal wear of the cathode button and ring anode and suggest operational lifetimes compatible with a wide variety of space mission scenarios. The current test series utilized a sacrificial graphite nozzle insert, as illustrated in Fig. 17, for ease of use and as a cost saving measure. Despite the well known aggressive erosive action of hydrogen on carbon at high temperature, ${ }^{17}$ we found the throat erosion rate to be within acceptable limits for performance evaluation studies. Previous attempts to utilize graphite constrictor/anode nozzle assemblies in medium-power hydrogen arcjets encountered increasingly significant constrictor growth with increasing specific input power, ${ }^{18}$ but in our configuration the graphite nozzle does not need serve as a constrictor/anode and can therefore exhibit greater endurance and life. Carbon loss was clearly evident from afterburning radiative soot emissions from the plume, as revealed by a typical plume image in Fig. 18, but periodic measurements of throat diameter indicated very moderate growth rates over the course of the test program. 


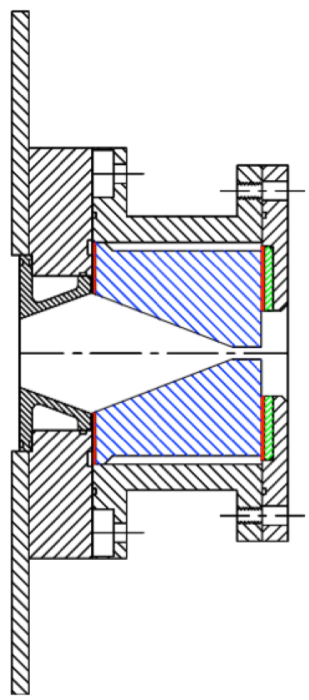

Figure 17. Graphite nozzle design detail.

When testing with hydrogen at the highest levels of specific input energy, however, heat loading on the nozzle insert generated extremely large thermal stresses at the throat and sometimes resulted in fracturing of the nozzle. This occured on three separate occasions and in all cases resulted in a symmetric crack pattern as shown in Fig. 19. It is believed that these high thermal stresses were related to the thickness of the part which caused an inordinately large radial temperature gradient between the throat surface and the back surface. It is suggested that this engineering flaw could be eliminated via a redesign that better accommodates the heat loading and relieves the thermal stress. Thus, given the moderate erosive rates observed during the test program, it is further suggested that the potential utilization of graphite as a practical arcjet nozzle material continue to be explored.

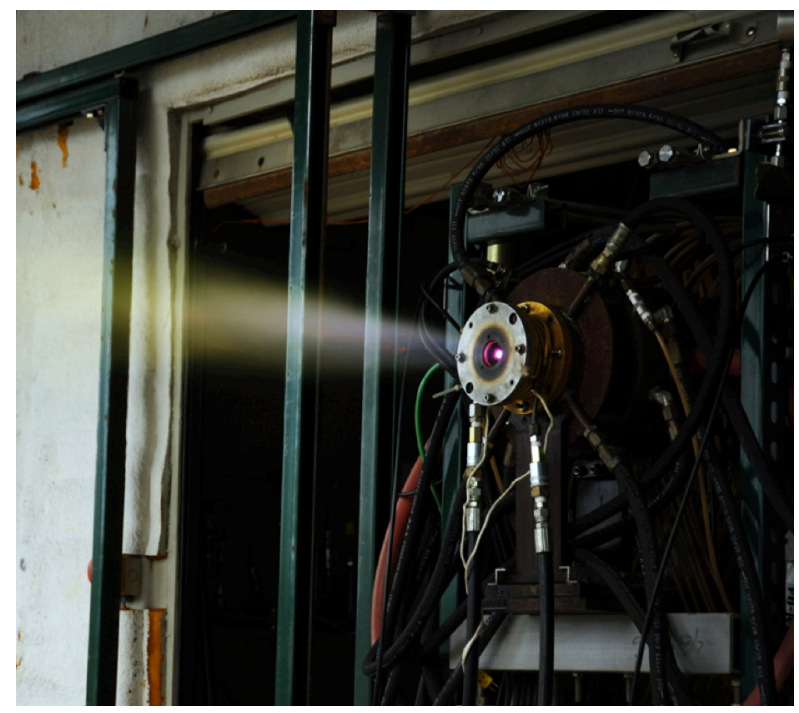

Figure 18. Typical hydrogen arcjet plume image.

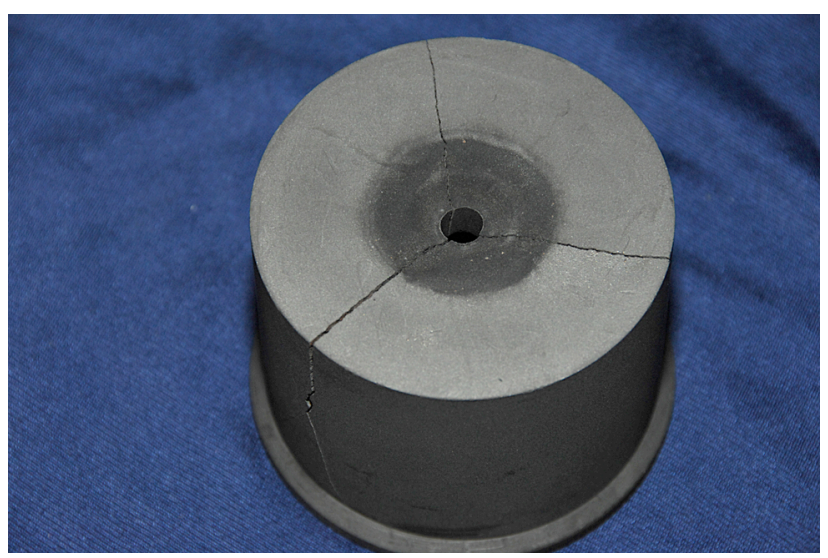

Figure 19. Fractured graphite nozzle insert.

\section{Conclusion}

Performance testing results for a MW-class breadboard flex-propellant arcjet represent a significant milestone achievement in the technical progression of regeneratively-cooled electrothermal plasma rockets. Although device configuration departs significantly from the modern compact design architecture through incorporation of a mixing plenum downstream of the anode attachment point and upstream of the nozzle throat, the approach benefits from attainment of EST conditions and may not be impractical with respect to regeneratively cooled thruster designs. Detailed analysis of current data infers good Isp and thrust efficiency performance up to a maximum specific input energy of $250 \mathrm{MJ} / \mathrm{kg}$ for hydrogen and $60 \mathrm{MJ} / \mathrm{kg}$ for simulated ammonia. The promising results thus far suggest strong grounds for continued technology/engineering development efforts in the 0.5-1.0 MW power range. The efforts should strongly focus on technical issues associated with regenerative cooling to include quantification of heat flux distribution, regenerative efficiency, and performance optimization and should begin addressing system and engineering design challenges leading toward the development and ground testing of a high-power flexpropellant arcjet thruster prototype.

\section{Acknowledgments}

The author thanks the NASA-MSFC Hot Gas Test Facility technical staff for assistance with arcjet setup and test operations support and Dr. Michael R. Lapointe, NASA-MSFC, for his encouragement and support of the research. 


\section{References}

${ }^{1}$ Todd, J. P., "30-kW Arcjet Thruster Research,” APL-TDR-64-58, Giannini Scientific Corp., Santa Ana, CA, Mar. 1964.

${ }^{2}$ John, R. R., Bennett, S., and Connors, J. F., “Arcjet Engine Performance: Experiment and Theory,” AIAA Journal, Vol. 1, No. 11, 1963, pp. 2517-2525.

${ }^{3}$ John, R. R., "Thirty Kilowatt Plasmajet Rocket Engine Development," RAD-SR-64-168, AVCO Corp., Wilmington, MA, 1964.

${ }^{4}$ Smith, W. W., Smith, R. D., Yano, S. E., Davies, K., and Lichtin, D., "Low Power Hydrazine Arcjet Flight Qualification," IEPC-1991-148, 1991.

${ }^{5}$ Butler, G. W., Cassady, R. J., and King, D. Q., “Directions for Arcjet Technology Development,” Proceedings of 25th AIAA Plasmadynamics and Lasers Conference, AIAA-1994-2652, June 1994.

${ }^{6}$ Bromaghim, D. R., LeDuc, J. R., Salasovich, R. M., Zimmerman, J. A., Matias, D. C., Sutton, A. M., Spanjers, G. G., Fife, J. M., Hargus, W. A., Spores, R. A., Johnson, L. K., Dulligan, M. J., Engelman, S. F., Schilling, J. H., and White, D. C., "An Overview of the On-Orbit Results from the ESEX Flight Experiment," Proceedings of 35th Joint Propulsion Conference, AIAA1999-2706, June 1999.

${ }^{7}$ Litchford, R. J. and Harada, N., "Multi-MW Closed Cycle MHD Nuclear Space Power via Nonequilibrium He/Xe Frozen Inert Plasma," Proceedings of Nuclear and Emerging Technologies for Space 2011, Paper 3349, American Nuclear Society, Albuquerque, NM, Feb. 2011.

${ }^{8}$ Haag, T. W. and Curran, F. M., "High-Power Hydrogen Arcjet Performance,” NASA TM-105143, 1991.

${ }^{9}$ Hoskins, W. A., Butler, G. W., and Kull, A. E., "A Comparison of Regenerative and Conventional Arcjet Performance," Proceedings of 30th Joint Propulsion Conference, AIAA-1994-3124, July 1994.

${ }^{10}$ Gölz, T. M., Auweter-Kurtz, M., and Kurtz, H. L., "100 kW Hydrogen Arcjet Thruster Experiments,” Proceedings of 28th Joint Propulsion Conference, AIAA-1992-3836, July 1992.

${ }^{11}$ Auweter-Kurtz, M., Glocker, B., Gölz, T., Kurtz, H. L., Messerschmid, E. W., Riehle, M., and Zube, D. M., “Arcjet Thruster Development," AIAA Journal of Propulsion and Power, Vol. 12, No. 6, 1996, pp. 1077-1083.

${ }^{12}$ Auweter-Kurtz, M., Gölz, T., Habiger, H., Hammer, F., Kurtz, H., Riehle, M., and Sleziona, C., "High-Power Hydrogen Arcjet Thrusters," AIAA Journal of Propulsion and Power, Vol. 14, No. 5, 1998, pp. 764-773.

${ }^{13}$ Gracey, C. M. and Jassowski, D. M., "Description of the Hyperthermal Environment Simulator (HES), A One-Megawatt Plasma Generator Facility,” Aerojet Nuclear Systems Company, Technical Rept. m001-TRU04-W236a2, Doc. No. N-2300, Sept. 1970.

${ }^{14}$ Painter, J. H., and Shaeffer, J. F., “Arc Heater Performance on Hydrogen, Helium, or Air,” AIAA Journal, Vol. 14, No. 9, 1976, pp. 1161-1162.

${ }^{15}$ Jahn, R. G., Physics of Electric Propulsion, McGraw-Hill, Inc. New York, 1968

${ }^{16}$ Park, C., Raiche II, G. A., Driver, D. M., Olejniczak, J., Terrazas-Salinas, I., Hightower, T. M., and Sakai, T., "Comparison of Enthalpy Determination Methods for Arc-Jet Facility," AIAA Journal of Thermophysics and Heat Transfer, Vol. 20, No. 4, 2006, pp. 672-679.

${ }^{17}$ Barletta, R. E., Vanier, P. E., Adams, J. W., and Svandrlik, J. F., "Carbon Erosion in Hydrogen - The Midband Problem Revisited," Proceedings of 10th Symposium on Space Nuclear Power and Propulsion, Vol. 271, 1993, pp. $245-249$.

${ }^{18}$ Riehle, M., Kurtz, H. L., Hammer, F., and Auweter-Kurtz, M., "Graphite Nozzle Performance Investigations with High Power Arcjets," Proceedings of 25th International Electric Propulsion Conference, IEPC-1997-085, July 1997. 